\title{
SPECTRUM OF DISEASES DIAGNOSED BY BONE MARROW EXAMINATION IN CENTRAL INDIA
}

Rajendra Kumar Nigam ${ }^{1}$,Reeni Malik ${ }^{2}$, Suhas Kothari³, Devendra Gour ${ }^{4}$, Atul Shrivastava5 ${ }^{5}$, Sharda Balani6, Rajnikant Ahirwar7 , Rubal Jain ${ }^{8}$

\section{HOW TO CITE THIS ARTICLE:}

Rajendra Kumar Nigam, Reeni Malik, Suhas Kothari, Devendra Gour, Atul Shrivastava, Sharda Balani, Rajnikant Ahirwar, Rubal Jain. "Spectrum of Diseases Diagnosed by Bone Marrow Examination in Central India".Journal of Evolution of Medical and Dental Sciences 2014; Vol. 3, Issue 02, January 13; Page: 326-337,

DOI: $10.14260 /$ jemds/2014/1829

ABSTRACT: BACKGROUND: Hematological diseases are quite frequent in population and mostly present with anemia. Although diagnosed mostly by clinical examination and simple investigations, bone marrow examination is confirmatory for the diagnosis. Bone marrow aspiration is simple and safe invasive procedure. Spectrum of hematological diseases in the developing countries is different from those observed in developed countries. OBJECTIVE: There is a lack of information about the exact relative prevalence of hematological disorders especially in Central India that's why this study was undertaken to correlate clinico-hematological and bone marrow findings. MATERIAL AND METHODS: It is a retrospective study of 345 cases who underwent bone marrow examination in Department Of Pathology, Gandhi Medical College, Bhopal. RESULTS: In our study age range of cases was 2-80 years with male: female ratio of 1.28:1. Most common diseases observed were megaloblastic anemia (41.81\%), dimorphic anemia (16.72\%), hypoplastic anemia (9.09\%), ITP $(8.36 \%)$ and infective pathology (4.72\%). In Hematological malignancies (20.28\%), most common were AML $(6.95 \%$ of overall \& $34.28 \%$ of malignant diseases $\%$ ) followed by ALL (4.92 \% overall and $24.28 \% \%$ of malignancies). 3 cases of Gaucher's disease were also reported.96.81\% patients were anemic while $44.92 \%$ were pancytopenic. Most common presenting symptoms were fever (44.92\%) and bleeding manifestations (30.14\%) which include ecchymosis, epistaxis, petechiae, melena, hematuria and hematemesis. Splenomegaly, hepatomegaly and lymphadenopathy were found in $35.65 \%, 21.15 \%$ and $14.78 \%$ of cases respectively. CONCLUSION: In our study final diagnosis could be made in majority of cases $(92.76 \%)$ by bone marrow examination, although having varied clinical presentations such as fever, bleeding manifestations, ascites, hepatomegaly, splenomegalyetc and usually associated with anemia / pancytopenia/ thrombocytopenia.All hematological cases must undergo bone marrow examination to conclude the exact underlying pathology for appropriate treatment.

KEYWORDS: Bone marrow aspiration, Disease pattern, Central India.

INTRODUCTION: Hematological disorders are quite frequent in population, most of the times diagnosis can be arrived at by detailed clinical examination and few simple investigations. Most of the disorders presented with anemia. However without bone marrow examination diagnosis is usually not confirmed. Bone marrow aspiration will yield diagnostic result in majority of cases 1 , 2.Although bone marrow aspiration and biopsy are an uncomfortable procedure for the patient and should be performed only when there is a clear clinical indication 3.Bone marrow aspiration examination is one of the most frequent and relatively safe invasive procedure. Though invasive it can be easily performed even in presence of thrombocytopenia with little or no risk of bleeding. 
The spectrum of hematological diseases is relatively different in the developing world than the developed countries ${ }^{4}$. Megaloblastic anemia is common non -malignant hematological disorder5, $60-80 \%$ of the world population is affected by IDA and is most common preventable nutritional anemia in the world $6,7,8$.

AIM: Aim of this study was to understand the pattern of diseases encountered in bone marrow aspiration and to correlate bone marrow aspiration findings with age, gender, clinical and peripheral blood findings.

MATERIAL AND METHODS: This was a retrospective study. We reviewed all cases of bone marrow examination reported in the Department of Pathology, Gandhi Medical College, Bhopal over a period of four years from 2009-2012.Patients' clinical details, the demographic data, clinical symptoms hematological parameters were taken from medical records, were reviewed and peripheral blood and bone marrow smears stained with Leishman stain were studied. Additional stain MPO /PAS were used whenever necessary. Final diagnosis was confirmed by bone marrow examination morphologically using criteria for diagnosis as per standard norms in the text.

Inclusion criteria-All cases coming for bone marrow examination. Indications were unexplained anemia, pancytopenia, thrombocytopenia, fever, hepatosplenomegaly or bleeding manifestations.

Aspirates with inadequate material or dry tap were excluded from the study.

Ethics-Study was approved by Institutional Ethical Committee.

RESULT: Total of 345 patients who had undergone bone marrow examination were studied which consisted of 194 males and 151 females with male: female ratio-1.28:1. The age of patients ranged from 1-80 years. We found 99 (28.69\%) cases in pediatric age group (0-14 years). Maximum cases 129 (37.39\%) were in age group 20-40 years, 48 (13.91\%) \& 20(5.79\%) cases were in the age group 41-60 and 61-80 years respectively

\begin{tabular}{|c|c|c|c|c|}
\hline S.No & Diagnosis & $\begin{array}{c}\text { No.of cases (\%) } \\
\text { (out of } 345 \text { total cases) }\end{array}$ & Male:Female & $\begin{array}{c}\text { Maximum cases } \\
\text { in age group }\end{array}$ \\
\hline 1. & Megaloblastic anemia & $115(33.33 \%)$ & $\begin{array}{c}72 / 43 \\
(1.67: 1)\end{array}$ & $\begin{array}{l}52 \text { cases- } \\
20-40 \text { years, } \\
24 \text { cases- } \\
0-14 \text { years }\end{array}$ \\
\hline 2. & Dimorphic anemia & $46(13.33 \%)$ & $\begin{array}{c}22 / 24 \\
(0.91: 1)\end{array}$ & $\begin{array}{l}19 \text { cases- } \\
20-40 \text { years } \\
12 \text { cases- } \\
0-14 \text { years }\end{array}$ \\
\hline 3. & Hypoplastic anemia & $25(7.25 \%)$ & $\begin{array}{c}3 / 12 \\
(1.08: 1)\end{array}$ & $\begin{array}{l}9 \text { cases- } \\
20-40 \text { years } \\
8 \text { cases- } \\
0-14 \text { years }\end{array}$ \\
\hline
\end{tabular}




\begin{tabular}{|c|c|c|c|l|}
\hline 4 & \multirow{2}{*}{ ITP } & $\begin{array}{c}23 . \\
(6.67 \%)\end{array}$ & $\begin{array}{l}11 / 12 \\
0.91: 1)\end{array}$ & $\begin{array}{l}10 \text { Cases- } \\
0 \text { cases- } \\
5 \text { cals, } \\
20-40 \text { years }\end{array}$ \\
\hline 5 & Infective pathology & $13(3.77 \%)$ & $\begin{array}{c}6 / 7 \\
0.85: 1)\end{array}$ & $\begin{array}{l}6 \text { cases- } \\
20-40 \text { years }\end{array}$ \\
\hline
\end{tabular}

\section{TABLE1: DISEASEPATTERN OF MOST COMMON DISEASES WITH AGE \& SEX DISTRIBUTION}

Other diseases were 7, 5, 4, 3 \& 2 cases of hypersplenism, megaloblastic anemia with eosinophilia, aplastic anemia, Gaucher's disease and CDA respectively. 1 case of CH syndrome, vWD, hemolytic anemia, Erythroid hyperplasia and one normal bone marrow was found.In 25(7.25\%) cases diagnosis could not be made due to laboratory constraints.

\begin{tabular}{|c|c|c|c|c|c|c|c|c|}
\hline S.NO & DISEASE & $\begin{array}{c}0-14 \\
\text { years }\end{array}$ & $\begin{array}{l}15-19 \\
\text { years }\end{array}$ & $\begin{array}{l}20-40 \\
\text { years }\end{array}$ & $\begin{array}{l}41-60 \\
\text { years }\end{array}$ & $\begin{array}{l}60-80 \\
\text { years }\end{array}$ & Total & M:F \\
\hline 1. & AML & 8 & 3 & 8 & 4 & 1 & 24 & $1: 2$ \\
\hline 2. & $\begin{array}{l}\text { CML-accelerated } \\
\text { phase }\end{array}$ & 0 & 0 & 2 & 0 & 0 & 2 & $1: 1$ \\
\hline 3. & MPD & 4 & 1 & 1 & 1 & 0 & 7 & $1: 6$ \\
\hline 4. & $\mathrm{PV}$ & 0 & 0 & 1 & 0 & 0 & 1 & $1: 0$ \\
\hline 5. & ET & 0 & 0 & 0 & 1 & 0 & 1 & $0: 1$ \\
\hline 6. & $\mathrm{PNH}$ & 0 & 1 & 0 & 0 & 0 & 1 & $1: 0$ \\
\hline 7. & MDS & 0 & 1 & 1 & 0 & 0 & 2 & $2: 0$ \\
\hline 8. & ALL & 11 & 1 & 5 & 0 & 0 & 17 & $3.2: 1$ \\
\hline 9. & NHL & 1 & 1 & 0 & 2 & 0 & 4 & $3: 1$ \\
\hline 10. & $\begin{array}{l}\text { Hodgkin's } \\
\text { Disease }\end{array}$ & 0 & 0 & 2 & 0 & 0 & 2 & $2: 0$ \\
\hline 11. & CLL & 0 & 0 & 0 & 1 & 0 & 1 & $1: 0$ \\
\hline 12. & MM & 0 & 0 & 1 & 2 & 3 & 6 & $5: 1$ \\
\hline 13. & Round cell tumor & 1 & 0 & 0 & 0 & 1 & 2 & $1: 1$ \\
\hline TOTAL & & 25 & 8 & 21 & 11 & 5 & 70 & \\
\hline
\end{tabular}

Table. 2: Distribution of Hematological Malignancies according to Age \& sex.

Out of 345 cases malignancy was found in 70(20.28\%) cases, out of which most common were AML 24 cases(6.95\% overall; 34.28\% of malignancies) followed by ALL 17 cases (4.92\% overall;24.28\% of malignancies), others were 7 (2.02\% overall; $10 \%$ of malignancies) and $6(1.73 \%$ overall;8.57\% of malignancies) and 4(2.89\% overall and5.7\% of malignancies) were MPD(not otherwise specified), MM and NHL respectively. 2 cases, each of Hodgkin's disease and Round cell tumor, and 1 case each of PV, ET, PNH \& CLL were found.

In ALL, maximum cases 64.7\%(11/17) were under 14 years and AML $33.33 \%(8 / 24)$ each were found in the age group of under $14 \& 20-40$ years. 
In AML male: female ratio 1.4:1; maximum cases 8 were observed in both $0-14$ and 20-40 years age groups followed by 3 cases each of AML M1 and unclassified AML, 2 cases of AML M6 and 1 each of AML M5 and AML M2E0. Most common were AML M3 and AML M7, maximum (4/5) each were in below 40 years age group.

Among MPD, all 6 cases were female in variable age groups and in MDS both cases were males below 40 years.

In lymphoid malignancies, male: female ratio was 3.25:1;maximum cases (11) were in 0-14 year's age group. Maximum cases in ALL were in ALL- L 2 (15 cases).

In Multiple myeloma, male: female ratio was 5:1 with maximum cases in above 40 years age group.

\begin{tabular}{|c|c|c|c|c|}
\hline S.No & Symptom & $\begin{array}{c}\text { No of cases out } \\
\text { of } 345(\%)\end{array}$ & $\begin{array}{c}\text { Most common diseases/ } \\
\% \& \text { No of cases }\end{array}$ & Other Diseases \\
\hline 1. & Fever & $155(44.92 \%)$ & $\begin{array}{l}\text { 100\% in CML, CLL, ETand } \\
\text { CH syndrome } \\
\text { 75\% AA \&hypersplenism } \\
\text { Overall leukemia } \\
48.48 \%(16 / 33) \\
58.82 \% \text {-ALL, } \\
50 \% \text {-AML } \\
56 \% \text {-HA } \\
47.82 \% \text {-Infective } \\
\text { pathology\& ITP, } \\
39.13 \% \text { MA, } \\
34.78 \% \text { DA }\end{array}$ & $\begin{array}{l}\text { Number of cases were too } \\
\text { low to draw any conclusion }\end{array}$ \\
\hline 2. & Ascites & $20(5.80 \%)$ & $\begin{array}{ll}\text { Dimorphic } & \text { anemia } \\
4 / 46(8.69 \%) & \end{array}$ & $\begin{array}{l}\text { Megaloblastic anemia } \\
5 / 115(4.34 \%)\end{array}$ \\
\hline 3. & Edema & $12(3.48 \%)$ & $\begin{array}{ll}\text { Dimorphic } & \text { anemia } \\
5 / 46(10.86 \%)) & \end{array}$ & $\begin{array}{l}\text { Megaloblastic anemia } \\
6 / 115(5.21 \%)\end{array}$ \\
\hline 4. & Pedal edema & $29(8.41 \%)$ & $\begin{array}{l}\text { Megaloblasticanemia } \\
18 / 115(6.95 \%)\end{array}$ & DA3/46(6.52\%) \\
\hline 5. & Facialedema & $11(3.19 \%)$ & $\begin{array}{l}\text { Megaloblastic anemia } \\
5 / 115(4.34 \%)\end{array}$ & DA $2 / 46(4.34 \%)$ \\
\hline 6. & $\begin{array}{l}\text { Visual } \\
\text { disturbances }\end{array}$ & $3(0.87 \%)$ & $\begin{array}{l}\text { DM } \\
1 / 46(2.17 \%)\end{array}$ & $\begin{array}{l}\text { MA } \\
2 / 115(1.73 \%)\end{array}$ \\
\hline 7. & Gall stones & $6(1.74 \%)$ & $\begin{array}{l}1 \text { each in } \\
\text { DM(2.17\%), } \\
\text { ITP( } 4.34 \%) \text {, } \\
\text { NHL }(25 \%) \%\end{array}$ & $\begin{array}{l}\text { MA } \\
2 / 115(1.73 \%)\end{array}$ \\
\hline 8. & Rash & $12(3.48 \%)$ & $\begin{array}{ll}\text { Hypoplastic } & \text { anemia } \\
5 / 25(20 \%), & \end{array}$ & ITP 4/23(17.39\%) \\
\hline 9. & Jaundice & $14(4.06 \%)$ & $\begin{array}{l}\text { Infective pathology } 2 / 13 \\
(15.38 \%)\end{array}$ & MA 8/115(6.95\%) \\
\hline
\end{tabular}


ORIGINAL ARTICLE

\begin{tabular}{|c|l|l|l|l|}
\hline 10. & Icterus & $10(2.90 \%)$ & $\begin{array}{l}\text { Megaloblastic anemia } \\
6 / 115(5.21 \%)\end{array}$ & DM2/46(4.34\%) \\
\hline 11. & Gall stones & $6(1.74 \%)$ & NHL $(25 \%)$, & $\begin{array}{l}\text { each in DM (2.17\%), ITP } \\
(4.34 \%), \text { MA 2/115(1.73\%) }\end{array}$ \\
\hline 12. & $\begin{array}{l}\text { Bleeding Per } \\
\text { Rectum }\end{array}$ & $3(0.87 \%)$ & DM 1/46(2.17. \%) & MA 2/115(1.73\%) \\
\hline 13. & Ecchymosis & $6(1.74 \%)$ & ITP 4/23(17.39\%) & HA2/25(8\%) \\
\hline 14. & Epistaxis & $31(8.99 \%)$ & ITP 6/23(26.08. \%)) & MA 9/115(7.82\%) \\
\hline 15. & Petechiae & $8(2.32 \%)$ & AA 1/4(25\%), & $\begin{array}{l}\text { Each ITP 3/23 (13.04\%), } \\
\text { HA 3/25(12\%) }\end{array}$ \\
\hline 16. & Gum Bleed & $16(4.64 \%)$ & ITP 5/23(21.73\%), & MA 4/115(3.47\%). \\
\hline 17. & Hematemesis & $13(3.77 \%)$ & ITP 5/23 $(21.73 \%)$ & MA 4/115(3.47\%) \\
\hline 18. & Hematuria & $9(2.61 \%)$ & L2 2/15(13.33\%) & $\begin{array}{l}\text { Each ITP2/23 (8.69\%), } \\
\text { MA4/115(3.47\%) }\end{array}$ \\
\hline 19. & Malena & $18(5.22 \%)$ & $\begin{array}{l}\text { Hypersplenism } \\
3 / 7(42.85 \%)\end{array}$ & $\begin{array}{l}\text { Each L2 3/15(20\%), } \\
\text { MA 6/115(5.21\%) }\end{array}$ \\
\hline
\end{tabular}

In our study, most common symptoms observed were fever, bleeding manifestations followed by edema/ascites.

Most common causes found among bleeding manifestations-for ecchymosis, gum bleed, hematemesis and epistaxis were ITP followed by MA, for petechiae were Aplastic anemia followed by ITP while for malena and hematuria were hypersplenism and ALL L2 respectively.

Lymphadenopathy was found in 51cases (14.78\%), (Generalized lymphadenopathy-15 cases and cervical \&mesenteric-9 cases each) for which most common cause was ALL. Splenomegaly (35.65\%)and hepatomegaly (21.15\%) were most common in MA followed by dimorphic anemia. Osteolytic lesions(2.89\%) were found in MM.

Pancytopenia was observed in 155 cases (44.92\%) cases.

Most common underlying diseases (with number of cases) were Megaloblastic anemia(67), Hypoplastic anemia(20), Dimorphic anemia(13), ALL(9), AML \& Infective pathology (8 each).Other causes were hypersplenism and ALL L2 (5each), Aplastic anemia(4), ITP and MDS(3 each)and one each of AML- M6, AML -M7, ALL - L1, Chediak Higashi syndrome, Congenital dyserythropoietic anemia, Pure red cell aplasia, Erythroid hyperplasia and Gaucher's disease.

Hematological parameters in our study were as follows: 


\begin{tabular}{|c|c|c|c|c|c|}
\hline \multicolumn{2}{|c|}{} & \multicolumn{3}{c|}{ Anemia } & \multicolumn{2}{c|}{ Normal } & Increased \\
\hline $\begin{array}{c}\text { Hb } \\
\text { (gm./dl) }\end{array}$ & $\mathbf{1 - 5}$ (severe) & $\begin{array}{c}\mathbf{5 - 8} \\
\text { (moderate) }\end{array}$ & $\mathbf{8 - 1 1 ( m i l d )}$ & $\mathbf{1 1 - 1 8}$ & $\mathbf{1 8 - 2 5}$ \\
\hline No. of cases & $153(44.34 \%)$ & $142(41.15 \%)$ & $39(11.30 \%)$ & 10 & \\
& $\mathrm{MA}$ & $\mathrm{DM}$ & ITP $17.39 \%$ & $(2.89 \%)$ & 1 \\
Most & $56.52 \%(65 / 115)$ & $50 \%(23 / 46)$ & $(4 / 23)$ & ITP & Polycythemia \\
common & $\mathrm{DM}$ & MA 35.65\% & MA $7.82 \%$ & $17.39 \%$ & Vera \\
cause & $47.82 \%(22 / 46)$ & $(41 / 115)$ & $(9 / 115)$ & $(4 / 23)$ & \\
\hline
\end{tabular}

TABLE 4: Distribution of cases according to severity of anemia with most common causes

\begin{tabular}{|c|c|c|c|c|}
\hline Diseases & $\begin{array}{c}\text { Severe } \\
\text { Hb<6 gm./dl } \\
\text { No of cases }\end{array}$ & $\begin{array}{c}\text { Moderate } \\
\text { Hb 6-8gm/dl } \\
\text { No of cases }\end{array}$ & $\begin{array}{c}\text { Mild } \\
\text { Hb8-11 gm./dl } \\
\text { No of cases }\end{array}$ & $\begin{array}{c}\text { Total } \\
\text { No of cases }\end{array}$ \\
\hline Megaloblastic anemia & 65 & 41 & 09 & 115 \\
\hline Dimorphic anemia & 22 & 23 & 01 & 46 \\
\hline Hypoplastic anemia & 16 & 19 & 00 & 25 \\
\hline
\end{tabular}

Table 5: Common diseases with number of cases according to severity of anemia

\begin{tabular}{|c|c|c|c|}
\hline s.no & TLC & $\begin{array}{c}\text { No of cases out } \\
\text { of } 345\end{array}$ & Most common cause in decreasing order \\
\hline 1. & $\begin{array}{l}<4,000 \\
\text { (decreased) }\end{array}$ & $186(53.91 \%)$ & $\begin{array}{l}\text { Hypoplastic anemia } 100 \%(25 / 25), \text { Infective } \\
\text { pathology } 69.23 \%(9 / 13), \text { MA } 64.34 \%(74 / 115) \text {, } \\
\text { >DM } 43.47 \%(20 / 46),>\text { L2 } 240 \%(6 / 15)\end{array}$ \\
\hline 2. & $\begin{array}{l}4,001-11,000 \\
\text { (normal) }\end{array}$ & $109(31.59 \%)$ & $\begin{array}{l}\text { ITP } 60.86 \%(14 / 23),>\text { DM50\%(23/46), }>\text { MA } \\
30.43 \%(35 / 115)\end{array}$ \\
\hline 3. & $\begin{array}{l}11,001-50,000 \\
\text { (moderately } \\
\text { increased) }\end{array}$ & $38(11.01 \%)$ & $\begin{array}{l}\text { MPD50\% (3/6), >33.33\% L2 (5/15), \& MM(2/6), } \\
>\text { ITP } 26.08 \%(6 / 23),>\text { DM } 6.52 \%(3 / 46), \text { MA5.21\% } \\
(6 / 115)\end{array}$ \\
\hline 4. & $\begin{array}{l}50,001-10,00 \\
000 \text { (severely } \\
\text { increased) }\end{array}$ & $12(3.47 \%)$ & $\begin{array}{l}\text { 100\% CLL }(1 / 1),>50 \% \text { M2 }(2 / 40), \text { L1 }(1 / 2),>20 \% \\
\text { M3 \&M7 (1/5)) andL2 (3/15). }\end{array}$ \\
\hline
\end{tabular}

Most common causes for leucopenia were hypoplastic anemia, megaloblastic anemia \& infective pathology, for normal count were ITP \& Dimorphic anemia while for moderate leukocytosis were MPD \& MM, and for marked leukocytosis it was leukemia. 


\begin{tabular}{|c|c|c|c|}
\hline S.no & $\begin{array}{l}\text { Platelet count } \\
\text { (per } \mathbf{m m}^{3} \text { ) }\end{array}$ & $\begin{array}{c}\text { No of cases } \\
\text { out of } 345(\%)\end{array}$ & $\begin{array}{l}\text { Causes\%(no of cases out of } \\
\text { respective disease) }\end{array}$ \\
\hline 1. & $<50,000$ & $167(48.40 \%)$ & $\begin{array}{l}\text { 100\% -AA(4/4), MDS, M6, L1 (each 2/2) } \\
\text { M5, M2Eo(1/1), PV, PNH(1/1), } \\
\text { 80\% HA (20/25), AML M3 (4/5), } \\
\text { 75\%AML M2, NHL (3/4 each), } \\
\text { 73.33\% ALL L2(11/15), } \\
\text { 65.21\% ITP(15/23), } \\
\text { 60\% AML M7(3/5,) } \\
\text { 57.14\% hypersplenism(4/7), } \\
\text { 53.84\% Infective pathology(7/13), } \\
\text { 30.43\% DM }(14 / 46) .\end{array}$ \\
\hline 2. & $50,001-1,00,000$ & $86(24.92 \%)$ & $\begin{array}{l}\text { 100\%-PNH, vWD(each1/1), } \\
66.66 \% \text {-AML(2/3), } \\
42.85 \% \text {-Hypersplenism(3/7) } \\
30.43 \% \text { - MA(35/115), } \\
23.07 \% \text {-Infective pathology }(3 / 13) \text {, } \\
21.73 \% \text { - ITP, DM(5/23 \&10/46 } \\
\text { respectively) }\end{array}$ \\
\hline 3. & $\begin{array}{l}1,00,001-4,00,000 \\
\text { (normal range) }\end{array}$ & $89(25.79 \%)$ & $\begin{array}{l}\text { 66.66\% MPD(4/6), 47.82\% } \\
\mathrm{DM}(22 / 46), 24.34 \% \mathrm{MA}(28 / 115)\end{array}$ \\
\hline 4. & $4,00,001-20,00,000$ & $03(0.86 \%)$ & $\begin{array}{l}100 \% \text { ET(1/1), 50\%-CML in } \\
\text { accelerated phase(1/2), 20\% M7(1/5) }\end{array}$ \\
\hline
\end{tabular}

Most common causes for severe thrombocytopenia were Aplastic anemia, Leukemia \& Megaloblastic anemia, for moderate thrombocytopenia were PNH, AML \& hypersplenism, while for normal count were MPD \& DM, and for increased count were ET \& CML.

DISCUSSION: Bone marrow examination findings compared to other studies conducted were as follows-

\begin{tabular}{|c|l|l|l|l|l|l|}
\hline $\begin{array}{c}\text { Sl.n } \\
\text { o }\end{array}$ & & & & & & \\
\hline & \multirow{2}{*}{$\begin{array}{l}\text { STUDY/AUTHO } \\
\text { RS }\end{array}$} & $\begin{array}{l}\text { Pudasaini S. et al } \\
(2012)^{(9)}\end{array}$ & $\begin{array}{l}\text { Fazlur Raheem et } \\
\text { al (2005)(10) }\end{array}$ & $\begin{array}{l}\text { Anita } \\
\text { Tahlan } \\
\text { et.al(2008) } \\
(11)\end{array}$ & $\begin{array}{l}\text { Al-Ghazaly J } \\
\text { et al } \\
(2006)^{(12)}\end{array}$ & $\begin{array}{l}\text { Our study } \\
(2009-2012)\end{array}$ \\
\cline { 3 - 7 } & 57 & 424 & 742 & $\begin{array}{l}627 \text { patients } \\
\text { of } \\
\text { hematologic } \\
\text { al diseases } \\
\text { other than } \\
\text { lymphoma })\end{array}$ & 345 \\
\hline
\end{tabular}


ORIGINAL ARTICLE

\begin{tabular}{|c|c|c|c|c|c|c|}
\hline & $\begin{array}{l}\text { Age group } \\
\text { Maximum cases } \\
\text { in age group }\end{array}$ & $\begin{array}{l}9 \text { months }-75 \\
\text { years } \\
31-45 \text { years }\end{array}$ & $2-15$ years & $>15$ years & >14years & $\begin{array}{l}1-80 \\
\text { Years } \\
20-40 \text { years }\end{array}$ \\
\hline & Male:female & $1: 1.1(27: 30)$ & $1.96: 1(281: 143)$ & & $1.29: 1$ & $1.28: 1$ \\
\hline & $\begin{array}{l}\text { PERIPHERAL } \\
\text { BLOOD } \\
\text { FINDINGS- } \\
\text { Anemia } \\
\text { Pancytopenia } \\
\text { Bicytopenia } \\
\text { Thrombocytope } \\
\text { nia }\end{array}$ & $\begin{array}{l}86 \% \\
50 \% \\
36 \% \\
14 \%\end{array}$ & & & & $\begin{array}{l}96.81 \% \\
44.92 \% \\
34.23 \% \\
12 \%\end{array}$ \\
\hline & $\begin{array}{l}\text { BONE MARROW } \\
\text { DIAGNOSIS } \\
\text { NON } \\
\text { MALIGNANT }\end{array}$ & & & $72 \%(609)$ & & $79.72 \%(275)$ \\
\hline 1. & $\begin{array}{l}\text { Megaloblastic } \\
\text { anemia }\end{array}$ & $12.3 \%(7)$ & $24.92 \%(103)$ & $87 \%$ & & $\begin{array}{l}41.81 \% \\
(115)\end{array}$ \\
\hline 2. & $\begin{array}{l}\text { Pernicious } \\
\text { anemia }\end{array}$ & & & & $0.74 \%(3)$ & \\
\hline 3 & $\begin{array}{l}\text { Dimorphic } \\
\text { anemia/mixed }\end{array}$ & - & $4.7 \%(20)$ & & $2.87 \%(18)$ & $\begin{array}{l}16.72 \% \\
(46)\end{array}$ \\
\hline 4 & $\begin{array}{l}\text { Microcytic } \\
\text { anemia/IDA }\end{array}$ & $7 \%(4)$ & $3.53 \%(15)$ & & $10.36 \%(65)$ & \\
\hline 5 & MA with malaria & & & & $9.09 \%(57)$ & \\
\hline 6 & $\begin{array}{l}\text { With excess } \\
\text { eosinophils }\end{array}$ & & & $2.2 \%$ & & $1.81 \%(5)$ \\
\hline 7 & $\begin{array}{l}\text { Infective } \\
\text { pathology }\end{array}$ & $12.3 \%(7)$ & & $3 \%$ & & $4.72 \%(13)$ \\
\hline 8 & $\begin{array}{l}\text { Erythroid } \\
\text { hyperplasia }\end{array}$ & $21 \%(12)$ & & & & $0.36 \%(1)$ \\
\hline 9 & $\begin{array}{l}\text { ITP/Megakaryoc } \\
\text { ytic } \\
\text { thrombocytopen } \\
\text { ia }\end{array}$ & $10.5 \%(6)$ & $9.43 \%(40)$ & $2.9 \%$ & $4.62 \%(29)$ & $9.09 \%(23)$ \\
\hline 10 & $\begin{array}{l}\text { Hemolytic } \\
\text { anemia }\end{array}$ & & $0.70 \%(3)$ & & & $1.45 \%(1)$ \\
\hline 11 & $\begin{array}{l}\text { Hypoplastic } \\
\text { anemia }\end{array}$ & $5.3 \%(3)$ & & $2.6 \%$ & & $9.09 \%(25)$ \\
\hline 12 & AIHA & & & & $1.43 \%(9)$ & \\
\hline 13 & Aplastic anemia & & $14.15 \%(60)$ & & $5.74 \%(36)$ & $2.54 \%(4)$ \\
\hline 14 & $\begin{array}{l}\text { Malaria } \\
\text { falciparum }\end{array}$ & & $2.35 \%(10)$ & & & \\
\hline 15 & Leishmaniasis & $1.8 \%(1)$ & $5.98 \%(25)$ & & $1.75 \%(11)$ & \\
\hline 16 & Hypersplenism & - & $2.59 \%(11)$ & & & $2.03 \%(7)$ \\
\hline
\end{tabular}


ORIGINAL ARTICLE

Although study group was different in different studies- pediatric age group in Fazlur10, Anita $^{11} \& \mathrm{Al}$-Ghazaly ${ }^{12}$ as compared to our study where all age group was taken similar to Pudasaini S.et $\mathrm{al}^{9}$ all have reported male dominance.

PudasainiS ${ }^{9}$ reported anemia, pancytopenia, bicytopenia \& thrombocytopenia in $86 \%, 50 \%$, $36 \%$ and $14 \%$ cases respectively in comparison to $96.81 \%, 44.92 \%, 34.23 \%$ \& $12 \%$ respectively in anemia, pancytopenia, bicytopenia \& thrombocytopenia in our series.

Our findings are similar to others as majority of cases were in nonmalignant group and most common disease in nonmalignant group was megaloblastic anemia, Anita et al ${ }^{11}$ reported $87 \%$ MA unusually high, reason may be, she has studied all cases having anemia. In our series MA incidence is much higher $41.81 \%$ in comparison to $12.3 \%$ Pudasaini et al ${ }^{9}$ study in Nepal \& $24.92 \%$ reported by Fazlur Rahman et al 10, Pakistan, both are different geographically and in food behavior.

Dimorphic anemia in our study was $16.72 \%$ much higher than $4.7 \%$ in Fazlur Raheem et al 10 and $2.87 \%$ in AL-Ghazaly.J et al ${ }^{12}$ while Pudasaini $S$ et al $^{9}$ and Anita et al ${ }^{11}$ did not report it.

Hypoplastic anemia was $9.09 \%$ in our study higher than 5.3\% in Pudasaini S. et $\mathrm{al}^{9}$ and $2.6 \%$ in Anita et al11. 
ITP incidence in our study was $8.36 \%$ higher than $4.62 \%$ by AL-Ghazaly ${ }^{12}$ and $2.9 \%$ by Anita et al ${ }^{11}$ and lower than $10.5 \%$ by PudasainiS.et al $^{9}$ and $9.43 \%$ by Fazlur Raheem et al 10 .

Among infective pathology group, $4.72 \%$ was observed slightly higher than $3 \%$ reported by AL -Ghazaly et al ${ }^{12}$ and muchlower than $12.3 \%$ reported by Pudasaini S etal ${ }^{9}$.

Aplastic anemia was found in $1.45 \%$ in our series as compared to $14.15 \%$ by FuzlurRehman et al 10 and $5.74 \%$ by AL -Ghazaly et al 11 .

Megaloblastic anemia with eosinophiliawas reported $2.2 \%$ by Anita et al ${ }^{11}$ in contrast to our $1.81 \%$.

We found 3 cases of storage disorder -Gaucher's disease while it has not been reported by others.

Malaria was reported by AL -Ghazaly et al ${ }^{12}$ and Leishmaniasis was reported by Pudasaini et al ${ }^{9}$, Fuzlar et al ${ }^{10}$ and AL-Ghazaly ${ }^{12}$ while we have not found parasitic infestation.

Pernicious anemia was found by $\mathrm{Al}$ Gazalye ${ }^{12}$ alone, not by others as similar to our study.

Hematological malignancies constituted $20.28 \%$ of our cases higher than $18 \%$ reported by Anita Tahlan et al ${ }^{11}$, but lower than $27.12 \%$ by Fazlur Rahman et al ${ }^{10}$, and $47.48 \%$ by Al -Ghazaly J ${ }^{12}$

In our study, we observed 58.27\% acute leukemia (34.28\% AML, 24.28\% ALL), MPD 8.57 \%, MM 8.57\% and NHL $1.42 \%$ while Pudasaini S et al ${ }^{9}$ reported Acute leukemia 12.3\%, MDS 3.5\%\& MM 3.5\%, Fazlur Rahim et al10 found Acute leukemia 24.28\% (AML $6.36 \%$ \& ALL 17.92\%), Lymphoma $1.17 \%$, and $0.47 \%$ each CML \& Neuroblastoma. Anita et al ${ }^{11}$ also reported $41 \%$ Acute Leukemia, CMPD 30\%, Lymphoma 14.2\%, CLL 7.5\% \& MM 7\%, Al-Ghazaly J12 found Acute leukemia in $37.31 \%$ (AML 25.35\%, ALL11.96\%), CLL 5.74\% \&MM 1.27\%.

We have not found HCL and metastasis in bone marrow while it was reported by Al-Ghazaly Jet al 12 .

This variation may be due to differences in the studied age groups \& population in different areas.

Number of cases of IDA, CML \&hemolytic anemia are very less in our study, as in other studies also because majority of IDA and mixed anemia, CML \& hemolytic anemia are diagnosed on smear examination \& needs other tests not bone marrow for confirmation of diagnosis.

CONCLUSION: Bone marrow examination is an important step to reach at confirmatory diagnosis of majority of hematological disorders. In our study, patients commonly presented with fever, bleeding manifestations and organomegaly. The most common nonmalignant diseases in decreasing order of frequencies were megaloblastic anemia, dimorphic anemia, hypoplastic anemia, ITP, Infective pathology and hematological malignancies. Among hematological malignancies, most common were AML followed by ALL.

Anemia is not a diagnosis but manifestation of an underlying disorder. Thus, even mild asymptomatic anemia should be investigated to ascertain the cause; if we wish to give appropriate treatment and bone marrow examination will clinch the diagnosis in majority of hematological diseases. Further proper documentation will definitely help in planning of health policies and National programs of our country. 
ACKNOWLEDGENT- We would like to extend our special thanks to Dr. Udyan Bajpai, Dr.Ashish Koshti, Dr.Ritu Jaipuria, Dr. Neha Banseria and technician Mr. K.P.Verma for their support and assistance.

\section{Abbreviations used:}

MA-Megaloblastic Anemia

CH-Chediak Higashi syndrome

IDA-Iron Deficiency Anemia

DM-Dimorphic Anemia

CDA- Congenital Dyserythropoietic Anemia

ITP- Immune Thrombocytopenia

AA-Aplastic Anemia

HA-Hypoplastic Anemia

MM-Multiple Myeloma

vWD-Von Willebrand Disease

\section{REFERENCES:}

1. OnalIK, Sümer H, TufanA, ShorbagiA. "Bone marrow embolismafter marrow aspiration and biopsy”. Am J Hematol 2005.28; 78 (2):158.

2. Mussarat N, Raziq F. "The incidence of underlying pathology in pancytopenia. An experience of 89 cases". J of Post-graduate Medical Institute 2004; 18(1):76-9.

3. Bain BJ. "Bone marrow trephine biopsy”. J ClinPathol 2001; 54:737-42. (PUBMED).

4. Young NS. "Hematopoietic cell destruction by immune mechanisms in acquired aplasticanemia. SeminHematol 2000; 37: 3 -14.

5. Ng SC, Kuperan P, Chan KS, Bosco J, Chan GL."Megaloblasticanemia- a review from University Hospital, Kuala Lumpur". Ann Acad Med Singapore 1988;17:261-6.

6. Zlotkin S. "A new approach to control of anemia in "at risk" infants and children around the world". 2004 Ryley-Jeffs memorial lecture. Can J Diet Pract Res 2004; 65(3):136-8.

7. Andrew NC. "Medical progress: disorders of iron metabolism". N Eng J Med 1999; 341:198695.

8. IssaragrisilS, Leaverton PE, Chansung K, Thamprasit T, Porapakham Y, Young NS.The Aplastic Anemia Study Group: "The incidence of aplastic anemia in Thailand". Am J Hematol 1999; 61: $164-8$.

9. Pudasaini S, Prasad KBR, Rauniyar SK, Shrestha R, Gautam K, Pathak R, Koirala S, Manandhar U, Shrestha B. "Interpretation of bone marrow aspiration in hematological disorder". J of Pathology of Nepal 2012; 2: 309-312.

10. Fazlur Rahim, IrshadAhmad, Saiful Islam, Muhammad Hussain, Taj Ali Khan Khattak\&QudsiaBano. Spectrum of hematological disorders in children observed in 424 consecutive bone marrow aspirations/biopsies". Pakistan Journal of Medical Sciences 2005;21 No 4: 433-436.

11. Anita Tahlan, Cherry Bansal, AnshuPalta, Sandeep Chauhan. "Spectrum and analysis of bone marrow findings in anemic cases". Indian J Med Science, 2008; 62(8):336-339. 
12. Al-Ghazaly J, Al-Selwi AH, Abdullah M, Al-JahafiAK, Al-Dubai W, Al-HashdiA."Pattern of haematological diseases diagnosed by bone marrow examination in Yemen. A developing country experience. Clin Lab Haematol 2006; 28:376-81.

\section{AUTHORS:}

1. Rajendra Kumar Nigam

2. Reeni Malik

3. Suhas Kothari

4. Devendra Gour

5. Atul Shrivastava

6. Sharda Balani

7. Rajnikant Ahirwar

8. Rubal Jain

\section{PARTICULARS OF CONTRIBUTORS:}

1. Professor, Department of Pathology, Gandhi Medical College, Bhopal, Madhya Pradesh.

2. Professor and HOD, Department of Pathology, Gandhi Medical College, Bhopal, Madhya Pradesh.

3. Associate Professor, Department of Pathology, Gandhi Medical College, Bhopal, Madhya Pradesh.

4. Associate Professor, Department of Community Medicine, Gandhi Medical College, Bhopal, Madhya Pradesh.

5. Senior Research Officer, Population based Cancer Registry, ICMR, Department of Pathology, Gandhi Medical College, Bhopal, Madhya Pradesh.
6. Assistant Professor, Department of Pathology, Gandhi Medical College, Bhopal, Madhya Pradesh.

7. P G Student, Department of Pathology Studying, Gandhi Medical College, Bhopal, Madhya Pradesh.

8. P G Student, Department of Pathology Studying, Gandhi Medical College, Bhopal, Madhya Pradesh.

\section{NAME ADDRESS EMAIL ID OF THE CORRESPONDING AUTHOR:}

Dr. Rajendra Kumar Nigam,

C-116, Shahpura,

Bhopal, Madhya Pradesh, India - 462039.

Email - dr.rajendranigam@gmail.com

Date of Submission: 10/12/2013.

Date of Peer Review: 11/12/2013.

Date of Acceptance: 27/12/2013.

Date of Publishing: 09/01/2014. 